\title{
Acute infection by SIV: massive immunological damage Mario Roederer*
}

Address: Chief, ImmunoTechnology Section, Vaccine Research Center, National Cancer Institute, Bethesda, MD, USA

* Corresponding author

from 2006 International Meeting of The Institute of Human Virology Baltimore, USA. 17-2I November, 2006

Published: 2I December 2006

Retrovirology 2006, 3(SuppI I):SI8 doi:I0.II86/I742-4690-3-SI-SI8

(C) 2006 Roederer; licensee BioMed Central Ltd.

Acute infection by SIV (and presumably HIV) does not cause a transient, mild insult to the immune system once thought. Rather, it is a fast and nearly catastrophic event, during which a vast majority of memory CD4 T cells are destroyed. Using careful quantitation of cell-associated viral load, together with precise identification of subsets, we showed that upwards of $80 \%$ of memory CD4 T cells were destroyed by SIV during the acute phase. This destruction could be solely ascribed to direct infection (no bystander mechanisms), and was evident throughout the body (i.e., not restricted to or even preferentially in the gut mucosa). We hypothesize that the extent of the destruction of the CD4 memory compartment during this phase is a key predictor of chronic sequelae: that a greater destruction heralds faster progression, greater morbidity, and a shorter lifespan. To test this, we have vaccinated nonhuman primates with a variety of vectors encoding SIV genes (DNA, recombinant adenovirus, or both). We showed that prior vaccination significantly tempered the extent of infection of CD4 cells during the acute phase of disease, and this directly predicts subsequent time to death. Therefore, we have identified a key mechanism leading to progressive disease in infected subjects. In addition, these findings point to the importance of reducing the cell-associated viral load during acute infection, through therapeutic or vaccination strategies. 